\title{
Der epidemiologische Schlüsselindikator „Drogenbezogene Todesfälle und Mortalität von Drogenkonsumentinnen und Drogenkonsumenten“ in der Europäischen Union und in Norwegen
}

M. Busch

\author{
The Epidemiological Key Indicator "Drug-Related Deaths and Mortaility Among \\ Drug Users" in the European Union and Norway
}

\section{Zusammenfassung}

Anliegen: Drogenbezogene Todesfälle spielen in der öffentlichen Diskussion zur Drogensituation eine wichtige Rolle, wobei aber oft unklar ist, auf welche Definitionen sich diese Diskussion bezieht. Vorliegender Aufsatz erläutert und diskutiert die von der Europäischen Beobachtungsstelle für Drogen und Drogensucht (EBDD) vorgenommene Definition von direkt drogenbezogenen und indirekt drogenbezogenen Todesfällen. Anhand des Beispiels Österreich werden die beiden in der Europäischen Union und Norwegen üblichen praktischen Vorgangsweisen zur Erstellung der Statistik der drogenbezogenen Todesfälle - über das allgemeine Todesursachenregister bzw. über spezielle, für drogenbezogene Todesfälle konzipierte Register - miteinander verglichen. Zum Abschluss erfolgt eine Gegenüberstellung und Diskussion der in den Ländern der Europäischen Union und Norwegen vorliegenden Statistiken der drogenbezogenen Todesfälle. Ergebnisse: Im von der EBDD definierten Schlüsselindikator „Drogenbezogene Todesfälle und Mortalität von Drogenkonsumentinnen und Drogenkonsumenten“ wird eine strikte Trennung in direkt drogenbezogene Todesfälle (= tödliche Überdosierung unter Beteiligung mindestens einer illegalen Droge) und indirekt drogenbezogene Todesfälle (Todesfälle als Langzeitfolge des Drogenkonsums, Unfälle unter Drogeneinfluss) vorgenommen. Statistiken drogenbezogener Todesfälle als Indikator für die aktuelle Drogensituation sollen sich ausschließlich auf direkt drogenbezogene Todesfälle beziehen. Zur Erfassung indirekt drogenbezogener Mortalität können Mortalitätskohortenstudien herangezogen werden. Die definitorischen Vorgaben der EBDD wurden in den meisten Ländern der EU und in Norwegen umgesetzt. Dennoch sind die zurzeit in den Ländern publizierten Zahlen drogenbezogener Todesfälle nur sehr eingeschränkt vergleichbar. Als Gründe dafür werden unter ande-

\section{Abstract}

Concern: Drug related deaths play a major role in public discussions concerning the drug situation, but often it is not clear on which definitions these discussions are based. This article presents and discusses the definitions of direct drug related deaths as well as indirect drug related deaths elaborated by the European Monitoring Centre for Drugs and Drug Addiction (EMCDDA). The two commonly used ways to count the number of drug related deaths in the European Union and Norway - extraction of drug related deaths from the General Mortality Register as well as collecting data within a Special Register for drug related deaths - are compared based on Austrian data. The numbers of drug related deaths in the different countries of the European Union and Norway are presented and discussed. Results: The epidemiological key indicator "Drug-related deaths and mortality among drug users" defined by EMCDDA discriminates strictly between direct drug related deaths (= death due to intoxications where at least one illicit drug was involved) and indirect drug related deaths (= death caused by long term effects of drug use or accidents under influence of drugs). Statistics of drug related deaths as an indicator for the actual drug situation should be based on direct drug related deaths exclusively. To collect data on indirect drug related deaths mortality-cohorts can be used. In most countries of the European Union and Norway the definitions of EMCDDA are implemented. Nevertheless the comparability of drug related death statistics between countries is very limited. In case of data collection using a Special Register, still existing differences in the definitions of drug related deaths and differences in regards to data completeness and in case of data extraction from General Mortality Registers differences of coding-procedures are discussed as some reasons for lack of compar-

Institutsangaben

REITOX Focal Point Austria, Gesundheit Österreich GmbH, Geschäftsbereich ÖBIG, Wien

Korrespondenzadresse

Dr. Martin Busch · Gesundheit Österreich GmbH, Geschäftsbereich ÖBIG · Stubenring 6 .

1010 Wien, Österreich · E-mail: busch@oebig.at

Bibliografie

Suchttherapie 2006; 7: 154 - 161 @ Georg Thieme Verlag KG Stuttgart · New York

DOI 10.1055/s-2006-927291

ISSN 1439-9903 
rem weiterhin bestehende Definitionsunterschiede, Unterschiede hinsichtlich Vollständigkeit der gemeldeten Fälle bei für drogenbezogene Todesfälle spezifischen Registern und eine unterschiedliche Kodierpraxis bei der Erstellung des allgemeinen Todesursachenregisters diskutiert. Schlussfolgerungen: Ein Vergleich der Zahlen der (direkt) drogenbezogenen Todesfälle zwischen den Ländern der EU bzw. Norwegen ist derzeit nur sehr eingeschränkt und die Interpretation der Statistiken drogenbezogener Todesfälle nur unter Berücksichtigung von Hintergrundwissen möglich. Weitere Vereinheitlichungsbemühungen hinsichtlich Definitionen und Kodier- bzw. Klassifikationspraxis sind notwendig. Die gegenwärtige Strategie der EBDD, im Rahmen von Statistiken drogenbezogener Todesfälle ausschließlich auf direkte drogenbezogene Todesfälle abzuzielen sowie Bestrebungen, die Rahmendefinition für drogenbezogene Todesfälle europaweit einheitlich zu gestalten, stellen jedoch in wesentlichen Bereichen ein sinnvolles und praktikables Konzept dar.

\section{Schlliisselwörter}

Drogentod · drogenbezogener Todesfall - suchtgiftbezogener Todesfall $\cdot$ Mortalität $\cdot$ Kohortenstudie ability. Conclusions: Comparability of numbers of drug related deaths between the countries of the European Union and Norway is very limited and the interpretation of drug related deaths statistics must take into account a lot of background information. Further efforts to standardize definitions and practice of coding and classification are necessary. The strategy of EMCDDA to focus on direct drug related deaths for statistics of drug related deaths and efforts to implement unique definition in Europe are important steps to arrive at a meaningful and practical concept of drug related deaths.

Key words

drug-related death $\cdot$ mortality $\cdot$ cohort-study

\section{Einleitung}

Substanzbezogene Todesfälle lassen sich grob in Todesfälle in Verbindung mit dem Konsum legaler und solche in Verbindung mit dem Konsum illegaler Substanzen (Drogen) einteilen. Diese Einteilung beruht nicht auf der Toxizität oder „Gefährlichkeit“ von Substanzen, sondern stellt eine gesellschaftliche Übereinkunft aufbauend auf Traditionen dar. Dennoch hat diese Unterscheidung einen wesentlichen Einfluss auf die gesellschaftliche und politische Aufmerksamkeit, die diesen Todesfällen jeweils entgegengebracht wird, und sie spiegelt sich auch in der Datenerfassung und in der wissenschaftlichen Forschungstradition wider. So beschäftigt sich beispielsweise die 1993 gegründete Europäische Beobachtungsstelle für Drogen und Drogensucht (EBDD) (englische Bezeichnung: European Monitoring Centre for Drugs and Drug Addiction - EMCDDA) ausschließlich mit illegalen Drogen. Zur Schätzung der Mortalität als Folge des Konsums von Nikotin und Alkohol liegt eine Reihe von qualitativ hochwertigen epidemiologischen Studien vor, wobei die Mortalität meist über die Methode der Bestimmung so genannter "kausal attribuierbarer Anteile“ („attributable fractions“ oder „aetiologic fractions“) erfolgt (siehe z.B. $[1,2]$ und weiter unten). Im Bereich der Mortalität aufgrund des Konsums illegaler Substanzen existieren nur wenige Arbeiten mit diesem epidemiologischen Ansatz. Dies ist einerseits mit unterschiedlichen Forschungstraditionen und andererseits mit Faktoren wie der geringen Prävalenz des Konsums illegaler Drogen und dem sozialen Stigma, dem dieser Konsum unterworfen ist, zu erklären [3]. Vorliegender Aufsatz fokussiert auf die derzeit in Europa gebräuchlichste Art der Erfassung von Todesfällen aufgrund des Konsums illegaler Substanzen in Form von Statistiken drogenbezogener Todesfälle. Diese Statistiken von Todesfällen im Zusammenhang mit dem Konsum illegaler Substanzen spielen in der öffentlichen Diskussion zur Drogensituation eine wichtige Rolle, wobei oft unklar ist, auf welche Definitionen sich diese Diskussion bezieht. Dies spiegelt sich bereits in der Nomenklatur wider. So wird beispielsweise von Drogentoten, Drogenopfern, Suchtmittel- toten, Substanztod, suchtgiftbezogenen oder drogenbezogenen Todesfällen gesprochen, wobei „drogenbezogene Todesfälle“ oder auf Englisch „drug related deaths“ die definitorische Übereinkunft auf europäischer Ebene am besten umschreibt. In Österreich werden drogenbezogene Todesfälle als suchtgiftbezogene Todesfälle bezeichnet und die beiden Begriffe synonym verwendet.

\section{Definitorische Abgrenzung von direkt und indirekt} drogenbezogenen Todesfällen und Mortalität von Drogenkonsumentinnen und Drogenkonsumenten

Prinzipiell lassen sich aus der Sicht der Europäischen Beobachtungsstelle für Drogen und Drogensucht (EBDD) zwei große Gruppen „drogenbezogener Todesfälle“ unterscheiden:

\section{Direkt drogenbezogene Todesfälle}

Nach Definition der EBDD sind darunter Todesfälle zu verstehen, die direkt durch den Konsum illegaler Drogen verursacht wurden: „The EMCDDA definition of drug-related death in the key indicator "Drug-related deaths and mortality among drug users“ refers to those deaths that are caused directly by the consumption of drugs of abuse. These deaths occur generally shortly after the consumption of the substance(s)" ([4], S. 6). Unter direkt drogenbezogenen Todesfällen sind damit tödliche Überdosierungen unter Beteiligung mindestens einer illegalen Droge zu verstehen.

Diese Definition umfasst sowohl beabsichtigte als auch unbeabsichtigte Überdosierungen. Der Einschluss von beabsichtigten Überdosierungen (Suiziden) ist dahingehend zu problematisieren, dass die Droge unter Umständen lediglich Mittel zum Zweck war und der Selbstmord mit anderen Methoden passiert wäre, wenn keine Droge vorhanden gewesen wäre. Seitens der EBDD werden Suizide durch Überdosierung jedoch grundsätzlich den direkt drogenbezogenen Todesfällen zugerechnet, da in der Praxis kaum feststellbar ist, ob es sich bei tödlichen Überdosierungen um Selbst- 
morde gehandelt hat oder nicht - es sei denn, es liegt ein Abschiedsbrief vor. In der österreichischen Statistik der suchtgiftbezogenen Todesfälle des Jahres 2005 war beispielsweise bei vier von 191 tödlichen Überdosierungen ein Abschiedsbrief vorhanden [5]. Auch ist selbstdestruktives und hoch riskantes Konsumverhalten oft Teil der Suchtproblematik und in diesem Sinne können solche Fälle durchaus auch als Indikator für die aktuelle Drogensituation angesehen werden. Eindeutig problematisch wird die Zuordnung von beabsichtigten Überdosierungen zu den direkt drogenbezogenen Todesfällen, wenn Personen, die kein Drogenproblem haben, sich illegale Drogen lediglich zum Zweck des Suizids beschaffen. Wenn solche Fälle in gehäufter Weise aufträten, würde dies zu einer unkontrollierbaren Verfälschung der Statistik führen. Allerdings wäre dafür eine leichte Verfügbarkeit von für Suizide geeigneten illegalen Drogen notwendig, eine Situation, die derzeit in den Ländern der EU kaum gegeben ist.

Unfälle unter Drogeneinfluss (z.B. Verkehrsunfälle) sind in der EBDD-Definition direkt drogenbezogener Todesfälle nicht inkludiert, obwohl eigentlich ein direkter Zusammenhang zum Drogenkonsum besteht [6] und der Tod wie bei der tödlichen Überdosierung kurzzeitig nach dem Konsum erfolgt. Dies erklärt sich daraus, dass der Kausalbezug zwischen Drogenkonsum und Unfall im Einzelfall nicht oder nur sehr schwierig erschlossen werden kann. Auch wären die Zahlen unvollständig, da derzeit nicht bei allen Unfällen toxikologische Analysen durchgeführt werden.

Ein zentrales Problem bei der Klassifizierung von direkt drogenbezogenen Todesfällen ist der Umstand, dass bei tödlichen Überdosierungen in der Regel mehrere Substanzen, oft legale und illegale Substanzen gemischt, festgestellt werden. Beispielsweise wurden in Österreich im Jahr 2005 nur bei 18\% der direkt suchtgiftbezogenen Todesfälle ausschließlich Opiate nachgewiesen. Bei 9\% der Fälle wurden zwei oder mehrere illegale Suchtgifte festgestellt und in $73 \%$ der Todesfälle eine Kombination aus illegalen Drogen und legalen psychoaktiven Substanzen (psychoaktives Medikament oder Alkohol). In 38\% der Fälle wurden neben Suchtgiften auch psychoaktive Medikamente, in 16\% auch Alkohol und zu 19\% sowohl Alkohol als auch psychoaktive Medikamente nachgewiesen (Tab.1). Dass Opiate allein oder in Kombination bei $98 \%$ aller direkt drogenbezogenen Todesfälle festgestellt wurden, stellt einen Beleg dafür dar, dass diese Substanz (in Kombination mit anderen Substanzen) am risikoreichsten ist [5]. Dennoch ist nicht ganz auszuschließen, dass ein Teil der illegalen Drogen zugeschriebenen Todesfälle ohne zusätzlichen Alkoholkonsum oder die Einnahme psychoaktiver Medikamente nicht passiert wäre, und damit Alkohol bzw. psychoaktiven Medikamenten in man- chen Fällen die Schuld oder zumindest eine Teilschuld am Tod zukommt. Wie Tab.1 zu entnehmen ist, übersteigt beispielsweise zwar der Blutalkoholwert in der österreichischen Statistik der suchtgiftbezogenen Todesfälle 2005 in keinem einzigen Fall den von Berzlanovich [7] für tödliche Alkoholüberdosierungen angegebenen Grenzwert von 3,5\%. Dennoch ist bei höheren Blutalkoholkonzentrationen in Kombination mit Drogen von einer sich wechselseitig verstärkenden Wirkung auszugehen und dem Alkohol damit zumindest in einem Teil der Fälle eine kausale Wirkung zuzuschreiben.

Die Erfassung direkt drogenbezogener Todesfälle hat aus Sicht der EBDD zum Ziel, einen Aspekt der aktuellen Drogensituation abzubilden. Direkt drogenbezogene Todesfälle können in Zusammenschau mit den anderen von der EBDD definierten epidemiologischen Schlüsselindikatoren „Prävalenz des Drogenkonsums und Konsummuster in der Allgemeinbevölkerung“, „Prävalenz des problematischen Drogenkonsums“, „drogenbezogene Infektionskrankheiten“ und „drogenbezogener Behandlungsbedarf“ zur Beschreibung der aktuellen Drogensituation herangezogen werden.

\section{Indirekt drogenbezogene Todesfälle}

Nach Definition der EBDD sind unter indirekt drogenbezogenen Todesfällen unter anderem Tod durch Krankheiten, bei denen ein Bezug zum Drogenkonsum angenommen wird (z.B. AIDS, wenn die HIV-Infektion durch intravenösen Drogenkonsum erfolgte), Unfälle unter Drogeneinfluss oder Selbstmorde von Drogenabhängigen (nicht durch Überdosierung) zu verstehen. Der kausale Bezug zwischen Drogenkonsum und Tod ist indirekt und beruht auf Schlussfolgerungen. Bei indirekt drogenbezogenen Todesfällen liegt der Drogenkonsum oft weit zurück (z.B. bei Personen, die an AIDS versterben und sich beim Drogenkonsum mit HIV infiziert haben). Ein Bezug zur aktuellen Drogensituation ist hier oft nicht gegeben. Daher können indirekt drogenbezogene Todesfälle nicht zur Beschreibung der aktuellen Drogensituation herangezogen werden und eine „Zählung“ solcher Fälle im Rahmen von Statistiken drogenbezogener Todesfälle macht wenig Sinn. Ein weiterer Grund, indirekt drogenbezogene Todesfälle nicht in die Statistik der drogenbezogenen Todesfälle zu inkludieren, ist, dass die Zuordnung zu indirekt drogenbezogenen Todesfällen in den meisten Fällen auf Schlussfolgerungen statt direkten Belegen beruht und auch der indirekte Kausalbezug zwischen Drogenkonsum und Todesfall nicht nachgewiesen werden kann. Weiteres sind die Zahlen sehr unvollständig, da zu vermuten ist, dass beispielsweise bei Tod infolge einer durch intravenösen (i.v.) Drogenkonsum verursachten Sekundärschädigung (z. B. Hepatitis) dieser Sachverhalt oft nicht

\begin{tabular}{|c|c|c|c|c|c|}
\hline \multirow{2}{*}{$\begin{array}{l}\text { Suchtgifte und psychoaktive } \\
\text { Medikamente }\end{array}$} & \multicolumn{4}{|c|}{ Blutalkoholwert in Promille } & \multirow[t]{2}{*}{ Summe } \\
\hline & $<0,1$ & $0,1-0,99$ & $1-1,99$ & $2-2,99$ & \\
\hline ausschließlich Opiate & $31(18,3 \%)$ & $4(2,4 \%)$ & $8(4,7 \%)$ & $2(1,2 \%)$ & $45(26,6 \%)$ \\
\hline Opiate mit anderen Suchtgiften & $12(7,1 \%)$ & $6(3,6 \%)$ & $2(1,2 \%)$ & $1(0,6 \%)$ & $21(12,4 \%)$ \\
\hline Opiate mit psychoaktiven Medikamenten & $51(30,2 \%)$ & $17(10,1 \%)$ & $6(3,6 \%)$ & $5(3,0 \%)$ & $79(46,7 \%)$ \\
\hline $\begin{array}{l}\text { Opiate mit anderen Suchtgiften und } \\
\text { psychoaktiven Medikamenten }\end{array}$ & $14(8,3 \%)$ & $3(1,8 \%)$ & $2(1,2 \%)$ & $1(0,6 \%)$ & $20(11,8 \%)$ \\
\hline Suchtgifte ohne Opiate & 0 & $3(1,8 \%)$ & 0 & 0 & $3(1,8 \%)$ \\
\hline $\begin{array}{l}\text { Suchtgifte ohne Opiate mit } \\
\text { psychoaktiven Medikamenten }\end{array}$ & $1(0,6 \%)$ & 0 & 0 & 0 & $1(0,6 \%)$ \\
\hline Summe & $109(64,5 \%)$ & $33(19,5 \%)$ & $18(10,7 \%)$ & $9(5,3 \%)$ & $169(100 \%)$ \\
\hline
\end{tabular}

Tab. 1 Direkt suchtgiftbezogene Todesfälle nach Substanzbeteiligung in Österreich 2005; Anmerkung: Die Tab. bezieht sich auf 169 der insgesamt 191 direkt suchtgiftbezogenen Todesfälle, von denen detaillierte toxikologische Untersuchungsergebnisse vorlagen. (Quelle: Gesundheit Österreich GmbH Geschäftsbereich ÖBIG 2006, eigene Berechnungen [8]) 
erkannt wird. Wesentlich validere Aussagen über die Langzeitfolgen des Drogenkonsums und über indirekte Kausalbezüge zwischen Drogenkonsum und Tod erlauben Mortalitätskohortenstudien.

\section{Mortalitätskohorten von Drogenkonsumentinnen und Drogenkonsumenten}

Bei Mortalitätskohorten wird die Sterblichkeit einer bestimmten Gruppe von Drogenabhängigen (z.B. alle Personen, die zwischen 1993 und 1995 eine Substitutionsbehandlung begonnen haben) über die Jahre hinweg beobachtet. Dies geschieht in der Regel über einen Datenabgleich mit dem allgemeinen Todesursachenregister (d.h. es wird versucht, über Namen und Geburtsdatum herauszufinden, ob die Person im allgemeinen Todesursachenregister als verstorben aufscheint). Ziel von Mortalitätskohorten ist es, die Gesamtmortalität von Drogenkonsumentinnen und Drogenkonsumenten zu eruieren (d.h. direkt drogenbezogene Todesfälle, indirekt drogenbezogene Todesfälle und natürliche Mortalität). Über die im allgemeinen Todesursachenregister aufscheinende Todesursache (in der Regel nach ICD 9 oder ICD 10 kodiert) lassen sich wichtige Schlussfolgerungen über die Langzeitfolgen des Drogenkonsums ziehen. Die Ergebnisse mehrerer Jahre Arbeit zur Vereinheitlichung des methodischen Vorgehens auf europäischer Ebene und die Ergebnisse von Kohortenstudien in neun Ländern wurden von der EBDD 2002 in einem Bericht zusammengefasst [9]. Mortalitätsstudien werden oft auch zur Evaluierung von therapeutischen Maßnahmen eingesetzt (z.B. [10-12]). Die Ergebnisse von Mortalitätskohortenstudien können auch zur Modellierung von „kausal attribuierbaren Anteilen“ („drugs attributable fractions“) herangezogen werden. Im altersstandardisierten Vergleich mit der Sterblichkeit der Gesamtbevölkerung kann herausgerechnet werden, wie viele der Personen infolge ihres Drogenkonsums vorzeitig gestorben sind. Darauf aufbauend kann analysiert werden, über welche Krankheiten (Mediatorvariablen) der Zusammenhang zwischen Drogenabhängigkeit und vorzeitigem Tod erklärbar ist. Möglichkeiten der Verwendung von „drugs attributable fractions“ für Analysen im illegalen Drogenbereich werden derzeit von der EBDD im Rahmen einer Arbeitsgruppe diskutiert. Ein Problem bei der Interpretation der Ergebnisse von Mortalitätskohorten stellt die Repräsentativität der Stichprobe dar. Da die verwendeten Kohorten in der Regel eine bestimmte (nicht für alle Personen mit Drogenproblemen repräsentative) Gruppe von Personen sind (z.B. Personen in Substitutionsbehandlung), sind die sich ergebenden Mortalitätsraten nur eingeschränkt auf alle Personen mit Drogenproblemen verallgemeinerbar. Manifestiert sich beispielsweise bei einer Kohorte, die über das Substitutionsbehandlungsregister rekrutiert wurde, eine stabilisierende Wirkung der Substitutionsbehandlung, wird die Mortalität systematisch unterschätzt. Sind besonders auffällige Drogenabhängige mit schlechter Prognose überrepräsentiert, wird der Effekt des Drogenkonsums auf die Mortalität systematisch überschätzt.

\section{EBDD-Richtlinien zur Erfassung direkt drogenbezogener Todesfälle}

In der EU existieren zwei Arten der Datenerfassung von direkt drogenbezogenen Todesfällen. In 11 Mitgliedsstaaten der EU und in Norwegen beruht die offizielle Zahl der „drug related deaths“ $(\mathrm{DRD}=$ direkt drogenbezogene Todesfälle) auf dem allgemeinen
Tab. 2 Extraktion von direkt drogenbezogenen Todesfällen aus dem General Mortality Register basierend auf ICD 9; Legende: DRD = drug related death (Quelle: European Monitoring Centre for Drugs and Drug Addiction 2002, eigene Übersetzung)

\begin{tabular}{|c|c|}
\hline DRD-Kategorisierung ${ }^{1}$ & ICD-9-Code(s) \\
\hline Drogenpsychosen & 292 \\
\hline Drogenabhängigkeit & $304.0,304.2-9$ \\
\hline Drogenmissbrauch ohne Abhängigkeit & $305.2-3,305.5-7,305.9$ \\
\hline Vergiftungen (Unfälle) & $\begin{array}{l}\mathrm{E} 850.0, \mathrm{E} 850.8^{2}, \mathrm{E} 854.1-2 \\
\mathrm{E} 855.2, \& \mathrm{E} 858.8^{2}\end{array}$ \\
\hline $\begin{array}{l}\text { Selbstmord und Selbstschädigung durch } \\
\text { Vergiftung }\end{array}$ & E950.0², E950.4² \\
\hline $\begin{array}{l}\text { Vergiftungen, unbestimmt ob unbe- } \\
\text { absichtigt oder vorsätzlich }\end{array}$ & $\mathrm{E} 980.0^{2}, \mathrm{E} 980.4^{2}$ \\
\hline
\end{tabular}

1 Die Kategorien beziehen sich jeweils auf die Substanzen Opiate, Kokain, Amphetamine und Derivate, Cannabis und Halluzinogene.

2 In Kombination mit N-Codes (N965.0 und/oder N968.5 und/oder N969.6 und/ oder N969.7).

Tab. 3 Extraktion von direkt drogenbezogenen Todesfällen aus dem General Mortality Register basierend auf ICD 10 (Quelle: European Monitoring Centre for Drugs and Drug Addiction 2002, eigene Übersetzung)

\begin{tabular}{ll|}
\hline zugrunde liegende Todesursache $^{1}$ & ICD-10-Codes \\
\hline psychische und Verhaltensstörungen & $\mathrm{F} 11-\mathrm{F} 12, \mathrm{~F} 14-\mathrm{F} 16, \mathrm{~F} 19$ \\
\hline akzidentelle Vergiftung & $\mathrm{X} 42^{2}, \mathrm{X} 41^{3}$ \\
\hline vorsätzliche Selbstvergiftung & $\mathrm{X} 62^{2}, \mathrm{X} 61^{3}$ \\
\hline Vergiftung, Umstände unbestimmt & $\mathrm{Y}^{2} 2^{2}, \mathrm{Y} 11^{3}$ \\
\hline
\end{tabular}

${ }^{1}$ Auf welche Substanzen sich die Todesursachen beziehen, ist den jeweiligen ICD-10-Codes zu entnehmen.

2 In Kombination mit T-Codes: T40.0-9.

3 In Kombination mit T-Code: T43.6.

Tab. 4 Definition von direkt drogenbezogenen Todesfällen im Special Register (Quelle: European Monitoring Centre for Drugs and Drug Addiction 2002, eigene Übersetzung)

\section{zugrunde liegende Todesursache Substanzen}

\begin{tabular}{l|l}
$\begin{array}{l}\text { Vergiftung als Unfall, Selbst- } \\
\text { mord, Mord oder unter unbe- } \\
\text { stimmten Umständen }\end{array}$ & $\begin{array}{l}\text { ausschließlich Opiate } \\
\text { ausschließlich Methadon } \\
\text { mehrere Substanzen }{ }^{1} \text { inklusive Opiate } \\
\text { mehrere Substanzen }{ }^{1} \text { exklusive Opiate } \\
\text { nicht näher spezifizierte Substanzen }\end{array}$ \\
\hline
\end{tabular}

${ }^{1}$ Mindestens eine der folgenden Substanzen muss festgestellt werden: Opiate, Amphetamine, Kokain, Crack, Cannabis, Halluzinogene, Lösungsmittel oder synthetische Designerdrogen,

2 Es werden Fälle eingeschlossen, bei denen angenommen wird, dass mindestens eine der folgende Substanzen beteiligt war: Opiate, Amphetamine, Kokain, Crack, Cannabis, Halluzinogene, Lösungsmittel oder synthetische Designerdrogen.

Todesursachenregister („General Mortality Register“ - GMR) und in 14 Staaten auf einem eigens für DRD konzipierten Spezialregister („Special Register“ - SR). In Österreich beruht das SR auf Verdachtsmeldungen der Polizei und anderer Stellen, die dann an- 
hand gerichtsmedizinischer Befunde verifiziert werden. In mehreren internationalen Projekten wurden von der EBDD Rahmendefinitionen zur Ermittlung der DRD erarbeitet. Diese Definitionen sind für die Extraktion aus dem GMR für ICD 9 in Tab. 2 dargestellt. Verstorbene mit den ICD-9-Todesursachen durch Drogen induzierte Psychose, Drogenabhängigkeit, Drogenmissbrauch ohne Abhängigkeit, nicht absichtlich herbeigeführte Intoxikation (Unfall), Selbstmord durch Drogen und tödliche Drogenüberdosis, bei der unklar ist, ob sie absichtlich oder unabsichtlich erfolgte, werden den DRD zugerechnet. Die einbezogenen Substanzen sind Opiate, Kokain, Amphetamine und deren Derivate, Cannabis und Halluzinogene. $\mathrm{Zu}$ beachten ist, dass es sich immer um die zugrunde liegende Todesursache („underlying cause of death“) handelt. Unter dem „underlying cause of death“ wird a) die Krankheit oder Verletzung, die den Ablauf der direkt zum Tode führenden Krankheitszustände auslöste, oder b) die Umstände des Unfalls oder der Gewalteinwirkung, die den tödlichen Ausgang verursachten, verstanden [13]. Da immer nur eine Todesursache angegeben werden kann (monokausale Todesursachenstatistik), sind indirekt drogenbezogene Todesfälle wie z.B. Personen, die an Langzeitfolgen des Drogenkonsums versterben (z. B. an einer Hepatitis C), in der Regel nicht inkludiert, weil der Drogenkonsum als vorangegangene Krankheit nicht am Totenschein vermerkt war oder nicht als „underlying cause of death“ kodiert wird. In Abstimmung mit der WHO wurden die ICD-Codes so ausgewählt, dass sie der Definition von direkt drogenbezogenen Todesfällen (siehe oben) weitestgehend entsprechen.

Die für ICD 9 erarbeiteten Richtlinien wurden von der EBDD für ICD 10 adaptiert (Tab. 3), wobei darauf geachtet wurde, die Definition möglichst kompatibel zu jener für ICD 9 zu gestalten.

Für die Datenerfassung im Special Register werden die in Tab. 4 dargestellten Kategorien herangezogen.

Vergleichbarkeit der Erfassung von direkt drogenbezogenen Todesfällen über Special Register (SR) und General Mortality Register (GMR) am Beispiel Österreich

Da die Datenerfassung in den EU-Staaten und Norwegen auf unterschiedlichen Datenquellen beruht, stellt sich die Frage, inwie- fern die Daten zu drogenbezogenen Todesfällen aus SR und GMR vergleichbar sind. In Österreich, wo aufgrund der Existenz eines landesweiten SR die Möglichkeit besteht, die Statistik der drogenbezogenen Todesfälle auf beide Arten zu erstellen, wurde eine Reihe von Vergleichsuntersuchungen und Case-Finding-Studien durchgeführt. Abb.1 zeigt die Entwicklung der Zahlen der direkt drogenbezogenen Todesfälle in beiden Registern seit 1989.

Es zeigt sich, dass bis zum Jahr 2001 in beiden Registern relativ ähnliche Zahlen dokumentiert werden, während die Jahre 2002 und 2003 erhebliche Diskrepanzen aufweisen. Im Jahr 2004 ist wieder eine Annäherung der beiden Werte zu beobachten. Der Beginn der Diskrepanzen fällt mit der Umstellung des GMR von ICD 9 auf ICD 10 zusammen. Eine genauere Analyse der Übereinstimmung der beiden Register bietet Abb. 2, die auf einer Case-Finding-Studie beruht. Dabei wurde untersucht, welche Todesursachen jene Personen, die im SR als drogenbezogene Todesfälle registriert sind, im GMR aufweisen. Werden Personen, die im SR als drogenbezogene Todesfälle registriert sind und im GMR aufgrund von Diskrepanzen bei der Namensschreibung nicht gefunden wurden, ausgeschlossen, so kann ausgesagt werden, dass 1998 81\%, $199985 \%, 200086 \%, 200179 \%$ und 2002 41\% auch im GMR als direkt drogenbezogene Todesfälle aufscheinen. Über die weitere Entwicklung kann nichts ausgesagt werden, da noch kein Case-Finding für die aktuelleren Jahre vorliegt.

Über Details der Diskrepanzen zwischen GMR und SR im Jahr 2002 gibt Tab. 5 Aufschluss. Hier zeigt sich, dass der Großteil der Todesfälle zwar auch im GMR als Intoxikationen aufscheinen, aber in der Kategorie „nicht näher bezeichnete Arzneimittel, Drogen und biologisch aktive Substanzen“, die laut EBDD nicht als direkt drogenbezogen zu klassifizieren sind.

Tab. 6 zeigt, dass sich die Kodierpraxis im GMR vom Jahr 2002 ins Jahr 2003 geändert hat. Die Anzahl der Kodierungen für Intoxikationen mit „nicht näher bezeichnete Arzneimittel, Drogen und biologisch aktive Substanzen" ist stark gesunken und die Anzahl der Codierungen für Opioidabhängigkeit ist gestiegen. Als Grund dafür wird von Statistik Austria ein detailgenaueres Ausfüllen der Totenscheine durch die beschauenden Ärzte und ein vermehrtes Nachfragen bei fehlenden Angaben auf dem Totenschein angegeben (Leitner, persönliche Mitteilung).

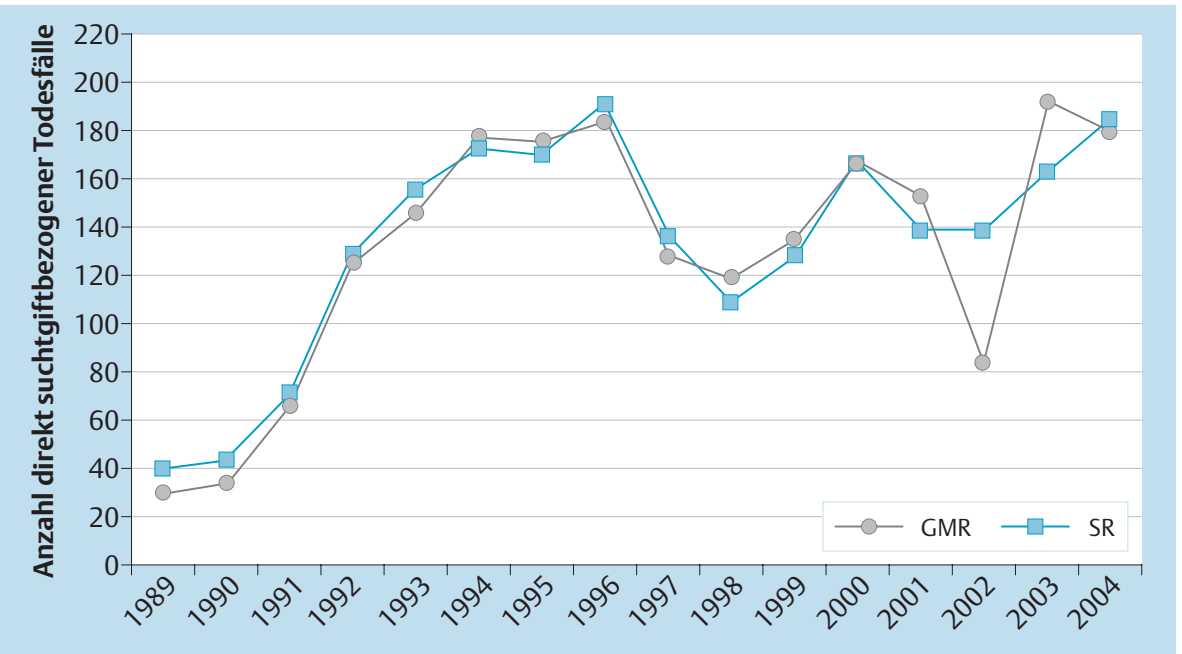

Abb. 1 Entwicklung der Anzahl der direkt drogenbezogenen Todesfälle in SR und GMR in Österreich, 1989 bis 2004; Legende: GMR = General Mortality Register, SR = Special Register (Quelle: Busch 2004 [8]). 


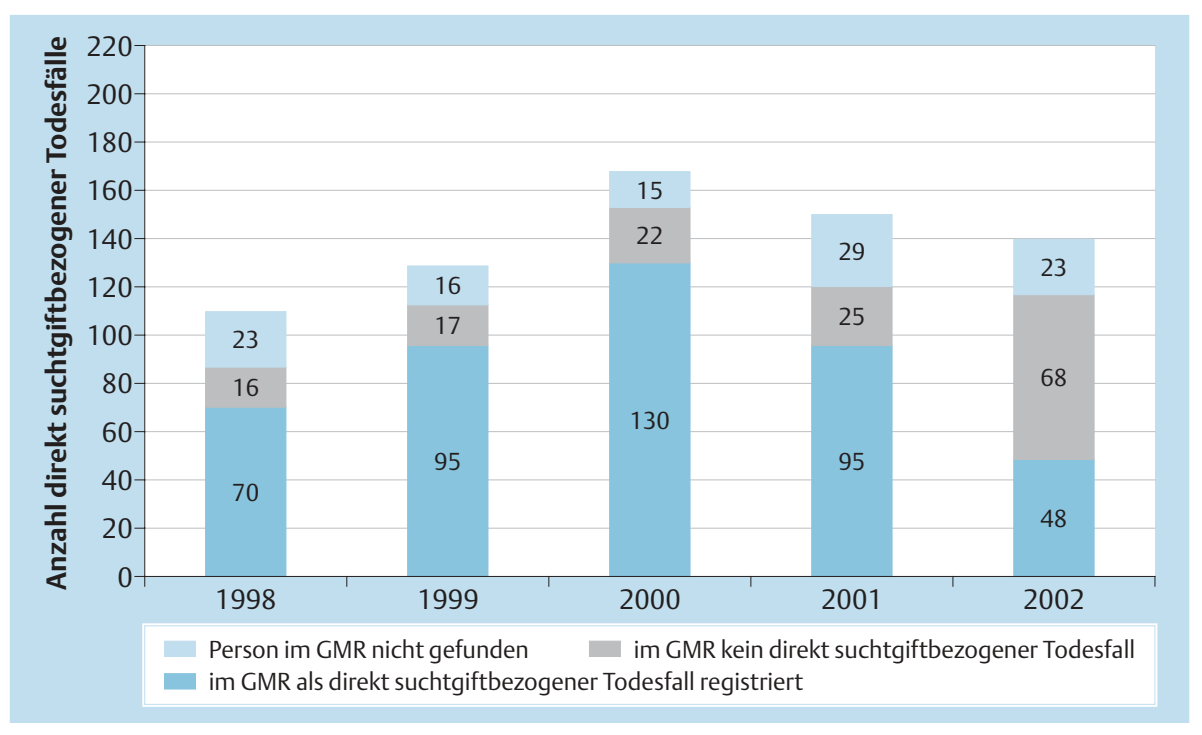

Abb. 2 Todesursachen von Personen im GMR, die im SR als direkt drogenbezogene Todesfälle registriert sind, in Österreich, 1998 bis 2002; Legende: $G M R=$ General Mortality Register, $\mathrm{SR}=$ Special Register (Quelle: Busch 2004 [8]).

Tab. 5 Häufigste ICD-10-Codes von Personen, die im SR als direkt suchtgiftbezogene Todesfälle aufscheinen und im GMR nicht als solche klassifiziert werden in Österreich, 2002 (Quelle: Busch 2004 [8])

\section{ICD-10-Code Häufigkeit Bedeutung}

\begin{tabular}{|ccl}
\hline X44, T509 & 25 & $\begin{array}{l}\text { akzidentelle Vergiftung durch sonstige nicht nä- } \\
\text { her bezeichnete Arzneimittel, Drogen und biolo- } \\
\text { gisch aktive Substanzen }\end{array}$ \\
\hline X64 T509 & 7 & $\begin{array}{l}\text { vorsätzliche Selbstvergiftung durch sonstige } \\
\text { nicht näher bezeichnete Arzneimittel, Drogen } \\
\text { und biologisch aktive Substanzen }\end{array}$ \\
Y14 T509 & 19 & $\begin{array}{l}\text { Vergiftung, Umstände unbestimmt durch sons- } \\
\text { tige nicht näher bezeichnete Arzneimittel, Dro- } \\
\text { gen und biologisch aktive Substanzen }\end{array}$ \\
\hline
\end{tabular}

Tab. 6 ICD-10-Codes für Opioidabhängigkeit und Vergiftungen mit „nicht näher bezeichneten Arzneimitteln, Drogen und biologisch aktiven Substanzen“ in Österreich, 2002 und 2003 (Quelle: Busch 2004 [8])

\begin{tabular}{|c|c|c|c|}
\hline ICD-10-Code & Bezeichnung & $\begin{array}{l}\text { Anzah } \\
2002\end{array}$ & 2003 \\
\hline F112 & Opioidabhängigkeit & 76 & 189 \\
\hline X44 T509 & $\begin{array}{l}\text { akzidentelle Vergiftung durch sonstige nicht } \\
\text { näher bezeichnete Arzneimittel, Drogen und } \\
\text { biologisch aktive Substanzen }\end{array}$ & 34 & 8 \\
\hline X64 T509 & $\begin{array}{l}\text { vorsätzliche Selbstvergiftung durch sonstige } \\
\text { nicht näher bezeichnete Arzneimittel, Drogen } \\
\text { und biologisch aktive Substanzen }\end{array}$ & 90 & 95 \\
\hline Y14 T509 & $\begin{array}{l}\text { Vergiftung, Umstände unbestimmt, durch } \\
\text { sonstige nicht näher bezeichnete Arzneimittel, } \\
\text { Drogen und biologisch aktive Substanzen }\end{array}$ & 47 & 22 \\
\hline
\end{tabular}

Zusammenfassend kann gesagt werden, dass GMR und SR in Österreich bis 2001 vergleichbare Zahlen zu den direkt drogenbezogenen Todesfällen geliefert haben. Mit der Einführung von ICD $10 \mathrm{im} \mathrm{Jahr} 2002$ lassen sich erhebliche Diskrepanzen feststellen, die aber durch eine Änderung der Kodierpraxis im GMR reduziert werden konnten. Ob diese Ergebnisse und insbesondere die Probleme im Zusammenhang mit der Einführung von ICD
10 auch auf andere EU-Länder, ganz besonders auf jene, die kein SR führen, übertragen werden können, ist zu diskutieren.

\section{Drogenbezogene Todesfälle im europäischen Vergleich}

In einigen Ländern, darunter auch in Österreich, wurde lange Zeit die Summe von direkt und indirekt drogenbezogenen Todesfällen als offizielle Zahl der „Drogenopfer“ präsentiert. Die intensive Auseinandersetzung mit der Thematik in internationalen Arbeitsgruppen auf Ebene der EBDD hat dann aber zur Entscheidung geführt, dass im Rahmen des Indikators „drogenbezogene Todesfälle" nur mehr direkt drogenbezogene Todesfälle erfasst werden sollen. Tab. 7 zeigt, dass die oben beschriebene Einschränkung der drogenbezogenen Todesfälle auf direkt drogenbezogene Todesfälle in allen Ländern der EU mit Ausnahme von Dänemark, Deutschland, Frankreich und Portugal umgesetzt wurde. In 17 Ländern entspricht die Zahl der direkt drogenbezogenen Todesfälle exakt der oben dargestellten EBDD-Definition.

Der in Tab. 7 dargestellte Vergleich der in den Ländern der EU und Norwegen berichteten Zahlen der drogenbezogenen Todesfälle zeigt erhebliche Unterschiede zwischen den Ländern. Während in den Ländern Frankreich, Lettland, Polen, Slowakei, Spanien und Ungarn sehr niedrige Zahlen (unter 10 Fälle pro Million Einwohnerinnen und Einwohner im Alter von 15 bis 64 Jahren) berichtet werden, liegen aus Dänemark, Luxemburg, Norwegen und dem Vereinigten Königreich Angaben von über 40 Fällen pro Million Einwohnerinnen und Einwohner im Alter von 15 bis 64 Jahren vor. In den restlichen Ländern liegen die Zahlen zwischen zehn und 40 Fällen pro Million. Werden die beiden extremsten Werte verglichen, so ergibt sich, dass in Dänemark die Zahl 43-mal so groß ist wie in Frankreich. Es stellt sich die Frage, worauf diese großen Unterschiede zwischen den Ländern zurückzuführen sind. Sie können sicher nicht ausschließlich durch tatsächliche Unterschiede in der Drogensituation erklärt werden. So liegen beispielsweise Schätzungen der Zahl der Personen mit problematischem Drogenkonsum für Deutschland zwischen 1,8 und 3,0 pro 1000 Personen der Altersgruppe 15 bis 64 und für Frankreich zwischen 3,8 und 4,8 pro 1000 Personen der Altersgruppe 15 bis 64 [14]. Dennoch werden in Deutschland auf eine 
Tab. 7 Anzahl (direkt) drogenbezogener Todesfälle absolut und pro einer Million Einwohnerinnen und Einwohner im Alter von 15 bis 64 Jahren in den Ländern der EU und Norwegen aus dem jeweils vorliegenden aktuellsten Jahr; Legende: GMR =General Mortality Register, $\mathrm{SR}=$ Special Register, EBDD = entspricht der Definition der Europäischen Beobachtungsstelle für Drogen und Drogensucht (Quelle: European Monitoring Centre for Drugs and Drug Addiction 2006 [14])

\begin{tabular}{|c|c|c|c|c|c|}
\hline Land & Quelle & Definition & Jahr & Anzahl & $\begin{array}{l}\text { Rate pro } \\
1000000 \\
15-\text { bis } \\
\text { 64-Jährige }\end{array}$ \\
\hline Belgien & GMR & EBDD & 1997 & 123 & 16,5 \\
\hline Dänemark & SR & $\begin{array}{l}\text { EBDD + Fälle mit star- } \\
\text { kem Kausalbezug } \\
\text { zwischen Drogenkon- } \\
\text { sum und Tod }\end{array}$ & 2004 & 275 & 76,7 \\
\hline Deutschland & SR & $\begin{array}{l}\text { EBDD + Tod aufgrund } \\
\text { der Langzeitfolgen } \\
\text { des Drogenkonsums } \\
+ \text { tödliche Unfälle un- } \\
\text { ter Drogeneinfluss }\end{array}$ & 2004 & 1385 & 24,5 \\
\hline Estland & GMR & EBDD & 2003 & 36 & 39,3 \\
\hline Finnland & GMR & EBDD & 2004 & 135 & 36,5 \\
\hline Frankreich & $S R$ & $\begin{array}{l}\text { EBDD + Überdosie- } \\
\text { rungen mit psycho- } \\
\text { aktiven Medikamen- } \\
\text { ten + Unfälle unter } \\
\text { Drogeneinfluss }\end{array}$ & 2004 & 69 & 1,8 \\
\hline Griechenland & SR & EBDD & 2004 & 240 & 32,1 \\
\hline Irland & GMR & EBDD & 2002 & 90 & 32,3 \\
\hline Italien & SR & EBDD & 2004 & 441 & 11,5 \\
\hline Lettland & GMR & EBDD & 2004 & 14 & 8,8 \\
\hline Litauen & GMR & EBDD & 2004 & 38 & 15,9 \\
\hline Luxemburg & $S R$ & EBDD & 2004 & 13 & 43,2 \\
\hline Malta & GMR & EBDD & 2004 & 6 & 18,4 \\
\hline Niederlande & GMR & EBDD & 2004 & 127 & 11,1 \\
\hline Norwegen & GMR & $\begin{array}{l}\text { EBDD ohne beabsich- } \\
\text { tigte Überdosierun- } \\
\text { gen }\end{array}$ & 2003 & 204 & 67,4 \\
\hline Österreich & SR & EBDD & 2004 & 185 & 33,0 \\
\hline Polen & GMR & $\begin{array}{l}\text { Es werden keine } \\
\text { T-Codes erfasst. F11, } \\
\text { F12, F14-16, F19, } \\
\text { X42, X62, Y12, X64, } \\
\text { Y14 }\end{array}$ & 2003 & 277 & 9,4 \\
\hline Portugal & SR & $\begin{array}{l}\text { alle Todesfälle, bei } \\
\text { denen ein positives } \\
\text { toxikologisches Er- } \\
\text { gebnis hinsichtlich ei- } \\
\text { ner illegalen Droge } \\
\text { vorliegt }\end{array}$ & 2004 & 156 & 18,1 \\
\hline Schweden & GMR & EBDD ohne T40.4 & 2003 & 152 & 25,7 \\
\hline Slowakei & $S R$ & EBDD & 2004 & 23 & 5,8 \\
\hline Slowenien & $S R+G M R$ & EBDD & 2004 & 40 & 27,8 \\
\hline Spanien & SR & EBDD & 2002 & 204 & 6,9 \\
\hline $\begin{array}{l}\text { Tschechische } \\
\text { Republik }\end{array}$ & SR & $\begin{array}{l}\text { EBDD + Überdosie- } \\
\text { rungen mit psycho- } \\
\text { aktiven Medikamen- } \\
\text { ten }\end{array}$ & 2004 & 241 & 29,5 \\
\hline Ungarn & SR & EBDD & 2004 & 34 & 4,9 \\
\hline $\begin{array}{l}\text { Vereinigtes } \\
\text { Königreich }\end{array}$ & GMR & $\begin{array}{l}\text { EBDD + Überdosie- } \\
\text { rungen mit psycho- } \\
\text { aktiven Medikamen- } \\
\text { ten }\end{array}$ & 2003 & 2964 & 67,9 \\
\hline Zypern & SR & EBDD & 2004 & 14 & 29,1 \\
\hline
\end{tabular}

Million Einwohnerinnen und Einwohner im Alter von 15 bis 64 Jahren bezogen 14-mal so viele drogenbezogene Todesfälle verzeichnet wie in Frankreich. Eine Ursache für die mangelnde Vergleichbarkeit zwischen den Ländern ist sicher in den trotz der Vereinheitlichungsbemühungen der EBDD immer noch bestehenden Unterschieden in der Definition von drogenbezogenen Todesfällen zu suchen. Definitionsunterschiede alleine können jedoch nur einen Teil der Unterschiede erklären. So ist beispielsweise die Definition von drogenbezogenen Todesfällen in Frankreich umfassender als die EBDD-Definition, dennoch sind die Zahlen extrem niedrig. Weitere wichtige Faktoren, welche die Zahl der direkt drogenbezogenen Todesfälle bei der Erfassung mittels SR beeinflussen, sind die Vollständigkeit der Meldung von Verdachtsfällen und bei der Erfassung mittels GMR mögliche Unterschiede in der Kodierpraxis bei der Feststellung der Todesursache. Diese Faktoren werden unter anderem von den organisatorischen Rahmenbedingungen der Datenerfassung und der gesellschaftlichen Aufmerksamkeit, die drogenbezogenen Todesfällen beigemessen wird, beeinflusst.

\section{Interpretation der Statistik der drogenbezogenen Todesfälle}

Auf die Unsinnigkeit der Aufsummierung von direkt und indirekt drogenbezogenen Todesfällen wurde bereits eingegangen. Möchte man drogenbezogene Todesfälle als einen Aspekt der aktuellen Drogensituation analysieren, so sind ausschließlich direkt drogenbezogene Todesfälle ist heranzuziehen. Ein Fehler, der bei der Interpretation der direkt drogenbezogenen Todesfälle in der Öffentlichkeit oft gemacht wird, ist, dass Veränderungen in der Statistik direkt als Veränderung der Drogensituation interpretiert werden. Unterschiede zwischen zwei aufeinanderfolgenden Jahren können aber auch einfach Zufallsschwankungen repräsentieren, die aufgrund der im statistischen Sinne geringen Anzahl der Fälle recht groß ausfallen können. Auf komplexe Zusammenhänge, wie z.B. die Tatsache, dass kurzfristig erfolgreiche Interventionen zunächst zu einer Verringerung der direkt drogenbezogenen Todesfälle führen, in späteren Jahren aber möglicherweise zu einem Anstieg (die suchtgiftabhängige Person überlebt aufgrund der kurzfristig erfolgreichen Intervention länger), geht [6] ausführlich ein. Kurzfristige Schwankungen der Zahl direkt drogenbezogener Todesfälle können auch durch lokale Gruppen von Personen mit Hochrisikodrogenkonsum verursacht werden. Bei der Analyse von Trends sind daher immer mehrere Jahre heranzuziehen und auch die Altersverteilung der drogenbezogenen Todesfälle ist zu beachten. Generell besteht immer die Gefahr, dass die Statistik der drogenbezogenen Todesfälle aufgrund ihrer „Dramatik“ bzw. des Fehlens anderer wichtiger Datenquellen in der Öffentlichkeit überinterpretiert wird. Im Rahmen einer seriösen Einschätzung der Drogensituation kann diese Statistik jedoch nur einen begrenzten Bereich abdecken und ist in Zusammenschau mit anderen epidemiologischen Schlüsselindikatoren zu interpretieren.

Ein Vergleich der Zahlen der (direkt) drogenbezogenen Todesfälle zwischen den Ländern der EU bzw. Norwegen ist derzeit nur sehr eingeschränkt und die Interpretation der Statistiken drogenbezogener Todesfälle nur unter Berücksichtigung von Hintergrundwissen möglich. Weitere Vereinheitlichungsbemühungen hinsichtlich Definitionen und Kodier- bzw. Klassifikationspraxis 
sind notwendig. Die gegenwärtige Strategie der EBDD, im Rahmen von Statistiken drogenbezogener Todesfälle ausschließlich auf direkte drogenbezogene Todesfälle abzuzielen sowie Bestrebungen, die Rahmendefinition für drogenbezogene Todesfälle europaweit einheitlich zu gestalten, stellen jedoch in wesentlichen Bereichen ein sinnvolles und praktikables Konzept dar.

\section{Literatur}

${ }^{1}$ Murray C, Ezzati M, Lopez A et al. Comparative quantification of health risks: conceptual framework and methodological issues (Chapter 1). In: Ezzati M, Lopez A, Rodgers A, Murray C (Hrsg). Comparative quantification of health risks: global and regional burden of disease due to selected major risk factors. Geneva: WHO, 2004

${ }^{2}$ Hoorn S, Ezzati M, Rodgers A et al. Estimating attributable burden of disease from exposure and hazard data (Chapter 25). In: Ezzati M, Lopez A, Rodgers A, Murray C (Hrsg). Comparative quantification of health risks: global and regional burden of disease due to selected major risk factors. Geneva: WHO, 2004

${ }^{3}$ English DR, Holman SDJ, Milne E et al. The quantification of drug caused morbidity and mortality in Australia 1995. Canberra, Australia: Commonwealth Department of Human Services, 1995

${ }^{4}$ European Monitoring Centre for Drugs and Drug Addiction. The DRDStandard 3.0 EMCDDA standard protocol for the EU member states to collect data and report figures on the key indicator drug-related deaths by the standard Reitox table. Lisbon: European Monitoring Centre for Drugs and Drug Addiction, 2002. http://www.emcdda.europa.eu/ ?fuseaction $=$ public. AttachmentDownload \&nNodeID $=1838$

${ }^{5}$ Gesundheit Österreich GmbH Geschäftsbereich ÖBIG. Suchtgiftbezogene Todesfälle 2005. Wien: Gesundheit Österreich GmbH Geschäftsbereich ÖBIG, 2006
${ }^{6}$ Uhl A. Was sind eigentlich ,Drogenopfer‘? In: Beubler E, Haltmayer H, Springer A (Hrsg). Opiatabhängigkeit, Interdisziplinäre Aspekte für die Praxis. Wien: Springer, 2003

${ }^{7}$ Berzlanovich A, Sofeit L, Muhm M et al. Alkoholintoxikationen - Eine retrospektive Analyse von Obduktionsfällen. Rechtsmedizin 1998; 8 (Suppl I): A34

${ }^{8}$ Busch M. Drug related death indicator in Austria. Unpublished Paper presented at the annual expert meeting on the key indicator drug related deaths in November 2004, Lisbon.

${ }^{9}$ European Monitoring Centre for Drugs and Drug Addiction. Mortality of drug users in the EU: coordination of implementation of new cohort studies, follow-up and analysis of existing cohorts and development of new methods and outputs. Lisbon: European Monitoring Centre for Drugs and Drug Addiction, 2002. http://www.emcdda.europa.eu/ ?fuseaction $=$ public. AttachmentDownload\&nNodeID $=1848$

${ }^{10}$ Brugal M, Domingo-Salvany A, Puig R et al. Evaluating the impact of methadone maintenance programmes on mortality due to overdose and aids in a cohort of heroin users in Spain. Addiction 2005; 100 (7): 981-998

${ }^{11}$ Esteban J, Gimeno C, Barril J et al. Survival study of opioid addicts in relation to its adherence to methadone maintenance treatment. Drug and Alcohol Dependence 2003; 70 (2): $193-200$

${ }^{12}$ Fugelstad A, Annell A, Rajs J et al. Mortality and causes and manner of death among drug addicts in Stockholm during the period 1981-1992. Acta Psychiatrica Scandinavica 1997; 96 (3): 169-175

${ }^{13}$ Statistik Austria. Todesursachen - Das Ausfüllen des Formblatts. Wien: Statistik Austria, 2006. http://www.statistik.at/fachbereich_03/totenbeschau/ausfuellen.shtml

${ }^{14}$ European Monitoring Centre for Drugs and Drug Addiction. EMCDDA statistical bulletin 2006. Lisbon: European Monitoring Centre for Drugs and Drug Addiction, 2006. http://www.emcdda.europa.eu 